\title{
Nrf2 activity as a potential biomarker for the pan-epigenetic anticancer agent, RRx-001
}

\author{
Shoucheng Ning ${ }^{1}$, Thillai Veerapazham Sekar ${ }^{2}$, Jan Scicinski ${ }^{4}$, Bryan Oronsky ${ }^{4}$, \\ Donna M. Peehl', Susan J. Knox ${ }^{1}$, Ramasamy Paulmurugan ${ }^{2}$ \\ ${ }^{1}$ Department of Radiation Oncology Stanford University Medical Center Stanford, CA 94305, USA \\ ${ }^{2}$ Department of Radiology Stanford University Medical Center Stanford, CA 94304, USA \\ ${ }^{3}$ Department of Urology Stanford University Medical Center Stanford, CA 94305, USA \\ ${ }^{4}$ EpicentRx, Inc., Mountain View, CA 94041, USA \\ Correspondence to: \\ Ramasamy Paulmurugan, e-mail: paulmur8@stanford.edu \\ Keywords: Nrf2, ARE, biomarker, oxidative stress, cancer
}

Received: March 23, $2015 \quad$ Accepted: May 21, $2015 \quad$ Published: June 04, 2015

\section{ABSTRACT}

Nuclear factor erythroid 2-related factor 2 (Nrf2) is a master regulatory transcription factor that plays an important role in the antioxidant response pathway against anticancer drug-induced cytotoxic effects. RRX-001 is a new anticancer agent that generates reactive oxygen and nitrogen species, and leads to epigenetic alterations in cancer cells. Here we report the RRx-001 mediated nuclear translocation of Nrf2 and the activation of expression of its downstream enzymes HO-1 and NQO1 in tumor cells. Inhibition of intrinsic Nrf2 expression by Nrf2-specific siRNA increased cell sensitivity to RRx-001. Molecular imaging of tumor cells co-expressing pARE-Firefly luciferase and PCMV-Renilla luciferase-mRFP in vitro and in vivo in mice revealed that RRx-001 significantly increased ARE-FLUC signal in cells in a dose- and time-dependent manner, suggesting that RRx-001 is an effective activator of the Nrf2-ARE signaling pathway. The pre-treatment level of ARE-FLUC signal in cells, reflecting basal activity of Nrf2, negatively correlated with the tumor response to RRX-001. The results support the concept that RRx-001 activates Nrf2-ARE antioxidant signaling pathways in tumor cells. Hence measurement of Nrf2-mediated activation of downstream target genes through ARE signaling may constitute a useful molecular biomarker for the early prediction of response to $\mathrm{RRx}-001$ treatment, and thereby guide therapeutic decision-making.

\section{INTRODUCTION}

Nuclear factor erythroid 2-related factor 2 (Nrf2) is a redox-sensitive master regulatory transcription factor that plays an important role in the antioxidant response pathway against chemotherapeutic drug-induced cytotoxic effects by oxidative stress [1]. Nrf2 in particular regulates the expression of antioxidant genes such as heme oxygenase 1 (HO-1), glutamate cysteine ligase catalytic subunit (GCLC), and $\mathrm{NAD}(\mathrm{P}) \mathrm{H}$ dehydrogenase quinone 1 (NQO-1), which neutralize intracellular accumulation of reactive oxygen species (ROS). Nrf2 protein level in cells is regulated by a cluster of inhibitory proteins, including Kelch-like ECH-associated protein 1 (Keap1) and Cullin 3 (Cul3) ubiquitin ligase. Keap1 represses Nrf2 activity by binding to its Neh2 domain and consequently promoting contact between $\mathrm{Nrf} 2$ and the $\mathrm{Cul} 3 / \mathrm{Rbx} 1$ ubiquitin ligase complex, leading to ubiquitination and degradation of Nrf2 by proteasomes [2]. Under normal conditions, Keap1 retains Nrf2 in the cytoplasm and ubiquitinylates Nrf2 to maintain its cellular threshold. Under oxidative stress, the Nrf2-Keap1 interaction is disrupted by the modification of Keap1 at cysteine 151 and protein kinase C-mediated phosphorylation of Nrf2 at serine 40 [3-4]. These modifications allow the release of Nrf2 from Keap1, resulting in the translocation of $\mathrm{Nrf} 2$ from cytoplasm to nucleus, where Nrf2 heterodimerizes with small Maf or Jun proteins and binds to the antioxidant response element (ARE) in the upstream UTR of promoter regions and initiates transcription of antioxidant genes [5-6]. Many studies have reported that Nrf2 and its downstream target genes are overexpressed in cancer cells, providing 
a growth advantage and contributing to therapeutic resistance against chemotherapy and radiotherapy through activation of antioxidant genes and neutralization of ROS accumulation [7].

RRx-001 is an aerospace-derived anticancer agent with reactive nitrogen species (RNS)-generating chemistry that leads to epigenetic alterations, such as DNA methylation and histone acetylation in cancer cells [8-13]. $\mathrm{RNS}$, a collective term that includes highly reactive species such as peroxynitrite $\left(\mathrm{ONOO}^{-}\right)$, nitrogen dioxide radical $\left(\bullet \mathrm{NO}_{2}\right)$, and other nitrogen oxides, are formed when nitric oxide (NO), which is abundantly induced by RRx-001 under hypoxic conditions, reacts with superoxide anion $\left(\mathrm{O}_{2}^{-}\right)$. In turn, RNS regulate DNA methyltransferases and histone deacetylases. The broad-spectrum epigenetic modulator activity of RRx-001 leads to resensitization of chemo- and radio-resistant tumor cells to therapeutic intervention, and is a focus of several ongoing clinical trials [13, 14]. Cell growth arrest induced by RRx-001 correlates with increased ROS/RNS production. Inhibition of ROS generation by $\mathrm{N}$-acetylcysteine attenuates the antiproliferative effects of RRx-001 [11]. These findings suggest a crucial role of ROS and RNS as effectors of RRx-001-induced pro-oxidant damage and epigenetic activity in cancer cells.

A Phase I clinical trial demonstrated encouraging therapeutic responses in a heavily pretreated, chemo- and radioresistant patient population with multiple types of cancers. RRx-001 monotherapy was well tolerated, with no dose-limiting toxicities [14]. Preliminary data from an ongoing randomized proof-of-concept Phase II study of RRx-001 vs. regorafenib suggest a positive trend in overall survival compared to regorafenib alone in patients with advanced colorectal cancer (Carter C. et al. Annals of Oncology. 2015; 26(suppl 2):ii4-ii5).

To date no predictive factor of response has been described for RRx-001. Herein, we report the results of molecular imaging studies in vitro and in mice bearing murine squamous cell carcinoma SCC VII tumors co-expressing ARE-Firefly luciferase reporter to measure ARE signaling while constitutive CMV-Renilla luciferasemRFP fluorescent protein measures cell viability in response to treatment with $\mathrm{RRx}-001$. The results suggest that Nrf2-ARE and its downstream gene expression may serve as a biomarker for predicting response of tumors to $\mathrm{RRx}-001$ treatment, and to select cancer patients who would be most likely to respond to and benefit from RRx-001 therapy.

\section{RESULTS}

\section{Endogenous Nrf2 expression in RRx-001-treated cells in vitro and in vivo}

Intracellular oxidative stress disrupts Nrf2-Keap1 binding, resulting in the release and translocation of Nrf2 from cytoplasm to nucleus, where Nrf2 binds to the ARE sequence in the 5'-UTR of antioxidant genes and initiates transcription (5-6). To investigate whether RRx-001-generated ROS and RNS activate endogenous Nrf2 in tumor cells, SCC VII cells were treated with $2 \mu \mathrm{M}$ or $5 \mu \mathrm{M}$ RRx-001 for 24 hours (h). Cells were lysed and cytoplasmic and nuclear proteins were isolated for immunoblot analysis. Results showed that the intensity of the cytoplasmic Nrf2 protein bands was not significantly different in cells with or without treatment of RRx-001 (Figure 1A). However, treatment with 2 or $5 \mu \mathrm{M}$ RRx-001 caused a 6-fold increase in the amount of nuclear $\mathrm{Nrf} 2$ protein compared to the baseline $(0 \mu \mathrm{M} R \mathrm{Rx}-001)$, indicating that RRx-001 activated endogenous Nrf2 and caused nuclear accumulation in SCC VII cells.

Next, we investigated RRx-001-induced activation of endogenous Nrf2 in SCC VII tumors in vivo. Mice bearing SCC VII tumors were treated with a single intravenous dose of $10 \mathrm{mg} / \mathrm{kg}$ RRx-001. Tumors were collected 24 or $48 \mathrm{~h}$ later and cytoplasmic and nuclear proteins were isolated for immunoblot assay. As shown in Figure 1B, the intensity of both cytoplasmic and nuclear Nrf2 proteins was much higher in RRx-001-treated tumors compared to that from control tumors without RRx-001 treatment (CTL). The cytoplasmic Nrf2 proteins were increased by $2.0-$ and 2.4- fold in tumors at $24 \mathrm{~h}$ and $48 \mathrm{~h}$, respectively, after a single dose of $10 \mathrm{mg} / \mathrm{kg} \mathrm{RRx}-001$ compared to control tumor without RRx-001 treatment. The nuclear Nrf2 level also increased to $\sim 1.6-$ and 2.3 - fold at $24 \mathrm{~h}$ and $48 \mathrm{~h}$, respectively, after $10 \mathrm{mg} / \mathrm{kg} \mathrm{RRx}-001$ treatment. These results indicate that treatment with RRx-001 not only facilitated nuclear translocation of $\mathrm{Nrf} 2$, but also upregulated endogenous $\mathrm{Nrf} 2$ expression in SCC VII tumors in mice.

To verify the effect of RRx-001 on Nrf2 activation, we assayed the expression of Nrf2 downstream antioxidant enzymes HO-1 and NQO1 in RRx-001 treated cells in vitro and in vivo in tumor-bearing mice. SCC VII cells were exposed to 0,2 , or $5 \mu \mathrm{M}$ of RRx-001 in growth medium for $8 \mathrm{~h}$ or $24 \mathrm{~h}$, and whole cell lysates were used to assay the HO-1 and NQO1 enzyme levels by Western blot. For the in vivo study, mice bearing SCC VII tumors were injected intravenously with one dose of either solvent or $10 \mathrm{mg} / \mathrm{kg}$ $\mathrm{RRx}-001$, and $\sim 8 \mathrm{~h}$ and $24 \mathrm{~h}$ later tumors were collected after whole animal perfusion with PBS. Whole tumor homogenates were used to assay the HO-1 and NQO1 protein levels by Western blot. As shown in Figure 1C, the levels of HO-1 and NQO1 in RRx-001 treated cells in vitro were significantly increased in a dose- and timedependent manner. HO-1 enzyme increased by $\sim 2$-fold after $8 \mathrm{~h}$ exposure, and then returned to the basal level at $24 \mathrm{~h}$. The NQO1 enzyme increased by 2 - to 4 -fold at $8 \mathrm{~h}$ and over 6-fold at $24 \mathrm{~h}$. The levels of HO-1 and NQO1 in RRx001 -treated tumors in mice were also increased by $13 \%$ and $20 \%$ for $\mathrm{HO}-1$, and $12 \%$ and $27 \%$ for NQO1 at $8 \mathrm{~h}$ and $24 \mathrm{~h}$, respectively, post-RRx-001 injection, compared to solvent control tumors ( $p<0.05$ for all RRx-001-treated tumors compared to control, $n=6$ per time point) (Figure 1D). 
$\mathbf{A}$
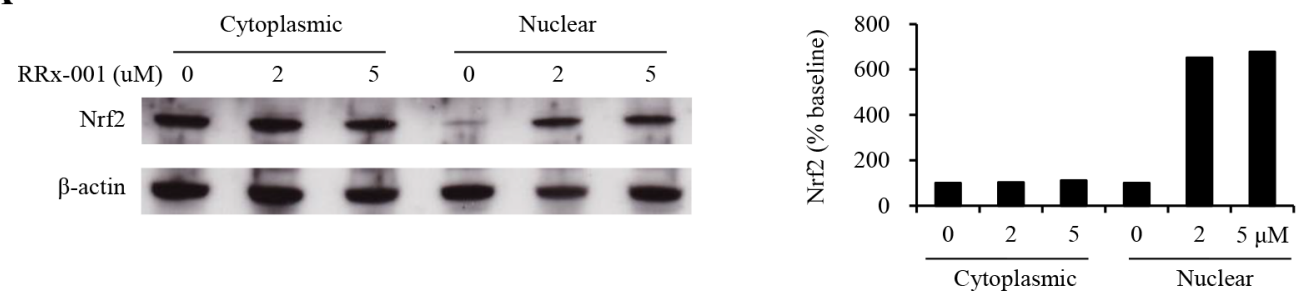

B
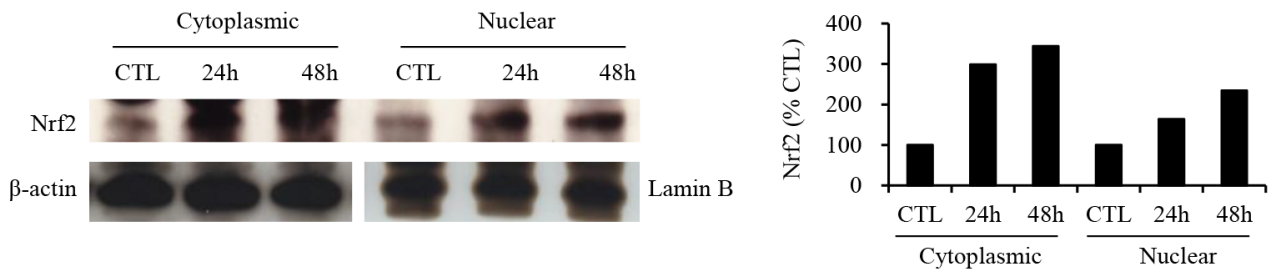

C
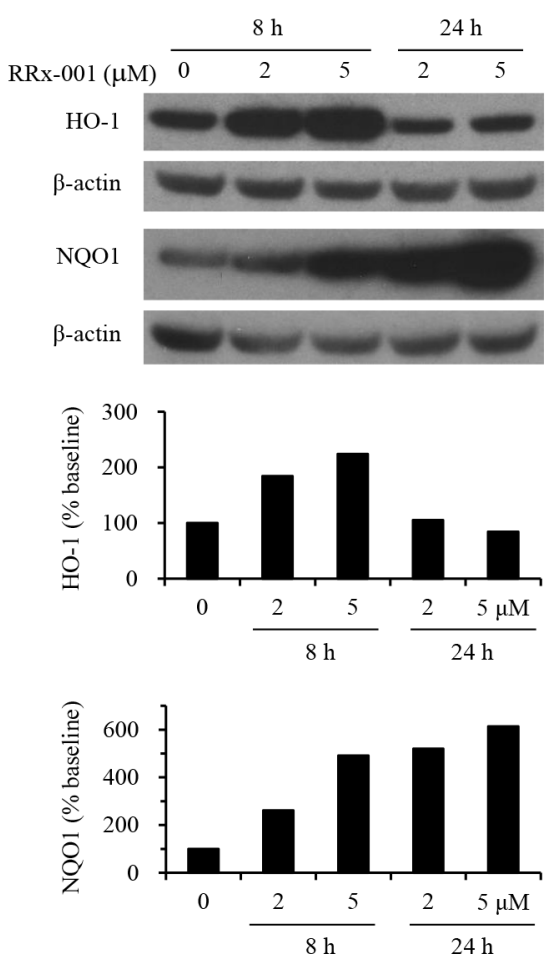

D
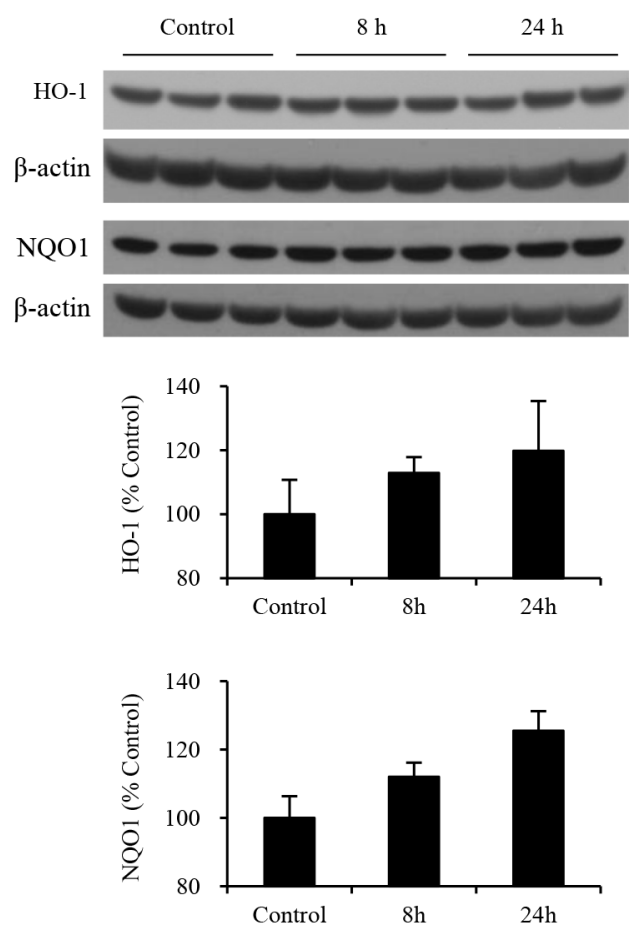

Figure 1: Nrf2, HO-1 and NQO1 expression in SCC VII tumor cells in vitro and in vivo. A. Nuclear and cytoplasmic Nrf2 levels in SCC VII cells in vitro. B. Nrf2 in tumors from mice treated with a single dose of solvent (CTL) or $10 \mathrm{mg} / \mathrm{kg}$ of RRx-001, and tumor samples were collected at $24 \mathrm{~h}$ or $48 \mathrm{~h}$ after RRx-001 injection. C. HO-1 and NQO1 expression in cells in vitro treated with 0 , 2 , and $5 \mu \mathrm{M}$ of RRx-001 for $8 \mathrm{~h}$ and $24 \mathrm{~h}$. D. HO-1 and NQO1 in tumors from mice treated with one dose of solvent (Control) or $10 \mathrm{mg} / \mathrm{kg}$ of RRx-001 and tumors were collected $8 \mathrm{~h}$ or $24 \mathrm{~h}$ post-treatment. There are three tumors per group presented in the Western blots, and the average levels of HO-1 and NQO1 of 6 tumors in each group were presented in the plots.

\section{Effect of RRx-001 in SCC VII cells with knockdown of endogenous Nrf2}

SCC VII cells were transiently transfected with either Nrf2 siRNA or scrambled siRNA, and Nrf2 expression and response to RRx-001 were assayed. As shown in Figure 2A, Nrf2 expression was significantly decreased in cells transfected with Nrf2 siRNA (Nrf2 C2 and $\mathrm{Nrf} 2 \mathrm{C} 3$ ). The protein level of $\mathrm{Nrf} 2$ was decreased by $40 \%$ compared to the level in cells transfected with scrambled siRNA (Ctl siRNA). We then analyzed the response of cells to $\mathrm{RRx}-001$ treatment by using a modified MTT cell proliferation assay (WST-8 assay). Cells transfected with Nrf2 siRNA (Nrf2 C2 and Nrf2 C3) were more sensitive to RRx-001 compared to parental cells (wild) or cells transfected with scrambled siRNA (Ctl siRNA), with $\mathrm{IC}_{50}$ of 1.09, 1.04, 0.74 , and $0.75 \mu \mathrm{M}$ for parental, scrambled siRNA and Nrf2 siRNA-transfected C2 and C3 cells, respectively (Figure 2B). 
A
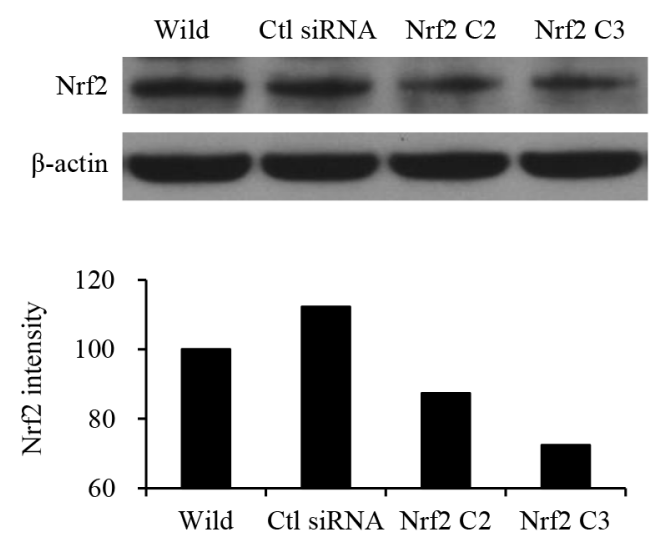

B

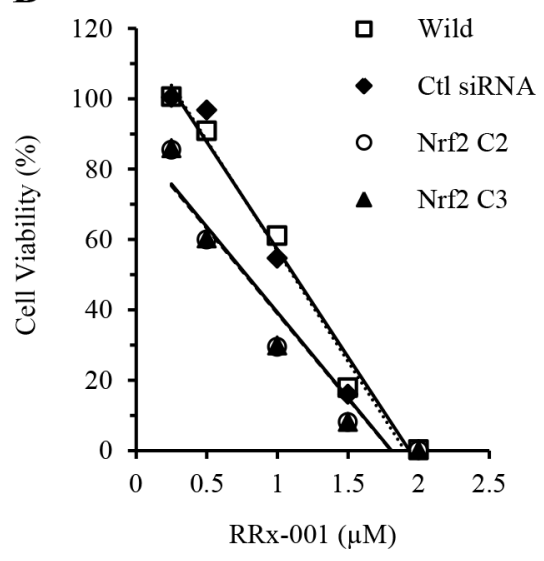

Figure 2: Effect of RRx-001 in SCC VII tumor cells transfected with Nrf2-specific siRNA. A. Western blot and quantitative graph showing the level of Nrf2 expression in cells transfected with control siRNA and Nrf2-specific siRNA. B. Cell viability assay in cells transfected with scrambled and Nrf2-specific siRNA in response to the treatment of different concentrations of RRx-001.

\section{RRx-001-mediated activation of ARE signaling in dual reporter gene transfected cells}

SCC VII tumor cells were stably co-transfected with ARE-Firefly luciferase (ARE-FLUC) pathway reporter gene and CMV-Renilla luciferase-monomeric red fluorescent protein (RLUC-mRFP). The ARE-FLUC reporter gene was used to measure ARE signaling, while the constitutive RLUC-mRFP signal was used to measure the cell viability in response to RRx-001 treatment. Positive clones were double selected by puromycin and G418, and further enriched by FACS, and verified for the expression of both reporter genes by a dual-luciferase reporter assay system. A single clone of cells co-expressing ARE-FLUC and CMV-RLUC-mRFP was expanded and used for evaluating ARE-FLUC response to RRx-001 treatment. The known Nrf2 activator TBHQ was used as a positive control in the study. The results showed that there was a dose- and time-dependent activation of ARE-FLUC reporter gene by RRx-001, with a maximum activation at $6 \mathrm{~h}$ post-treatment with $2.3 \mu \mathrm{M}$ of RRx-001 (Figure 3). The ARE-FLUC reporter signals gradually decreased at RRx-001 doses of 4.7-18.7 $\mu \mathrm{M}$, mainly due to the cell killing effect at these high doses of RRx-001 as indicated by decreased CMV-RLUC-mRFP signals. These results indicate that RRx-001 is an effective activator of the Nrf2-ARE pathway.

\section{Imaging of ARE reporter gene activation in $\mathrm{SCC}$ VII tumors in mice}

Mice bearing ARE-FLUC/RLUC-mRFP-expressing SCC VII tumors were treated with a single intravenous dose of $10 \mathrm{mg} / \mathrm{kg} \mathrm{RRx}-001$ and imaged for ARE-FLUC expression at $24 \mathrm{~h}$ before (pre-treatment) and $8 \mathrm{~h}$ and $24 \mathrm{~h}$ post-RRx-001 injection with a Xenogen IVIS 200 Imaging System. There were 10 mice (total of 20 tumors; 2 tumors per mouse) in each of RRx-001- and vehicle-treated groups. Figure 4 shows representative images of SCC VII tumors in mice with 5 animals/10 tumors for each group. The pre-treatment images were taken $24 \mathrm{~h}$ before RRx001 injection. The quantitation of ARE reporter gene signal was normalized by the RLUC-mRFP signal from the same tumor. There was a significant increase in the ARE-FLUC signals (5-fold higher compared to control) at $8 \mathrm{~h}$ following a single dose of RRx-001 treatment, and that was maintained at high levels until $24 \mathrm{~h}$, while there was no obvious change in the ARE-FLUC signals in the vehicle control-treated group. In a separate study, we treated mice with a lower dose of $5 \mathrm{mg} / \mathrm{kg} \mathrm{RRx}-001$ and found that the level of activation of ARE-FLUC signals was not significantly different from $10 \mathrm{mg} / \mathrm{kg}$ RRx-001 (Supplemental Figure 1).

\section{Correlation of ARE activity and tumor response to $R R x-001$ treatment}

Many studies have reported that the overexpression of Nrf2 and its downstream genes in cancer cells provides a survival benefit and growth advantage [7]. The Nrf2ARE signaling pathway is also involved in acquisition of resistance against anticancer therapies through activation of antioxidant genes and suppression of ROS accumulation and neutralization of electrophiles [7, 15-17]. We first evaluated the effect of co-expression of ARE-FLUC and CMV-RLUC-mRFP reporters on SCC VII tumor growth. Mice were subcutaneously inoculated with $5 \times 10^{5}$ cells of the parental and ARE-FLUC/ CMVRLUC-mRFP expressing SCC VII cells, and the tumor take rate and tumor size were monitored daily for 14 days. Results showed that the tumor take rates in syngeneic mice were $95 \%$ (19 tumors of 20 implanted) for both the parental and the ARE reporter-expressing tumor implants. 

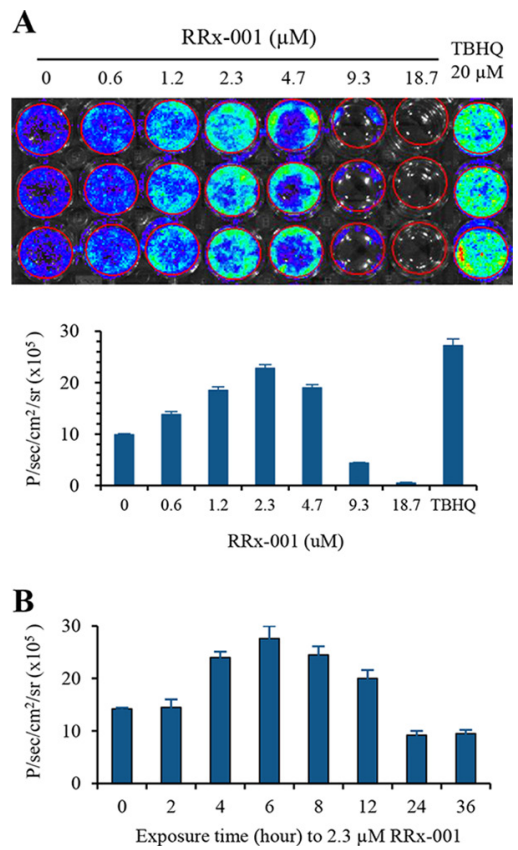

Figure 3: Reporter gene imaging to study the activation of ARE signaling by RRx-001 in SCC VII cells. A. Optical bioluminescence imaging and quantitative graph of ARE-FLUC signal in SCC VII cells stably expressing ARE-FLUC, $6 \mathrm{~h}$ after treatment with different doses of RRx-001. B. ARE-FLUC signal measured in SCC VII stable cells at different time points after treating with $2.3 \square \mathrm{M}$ of RRx-001.
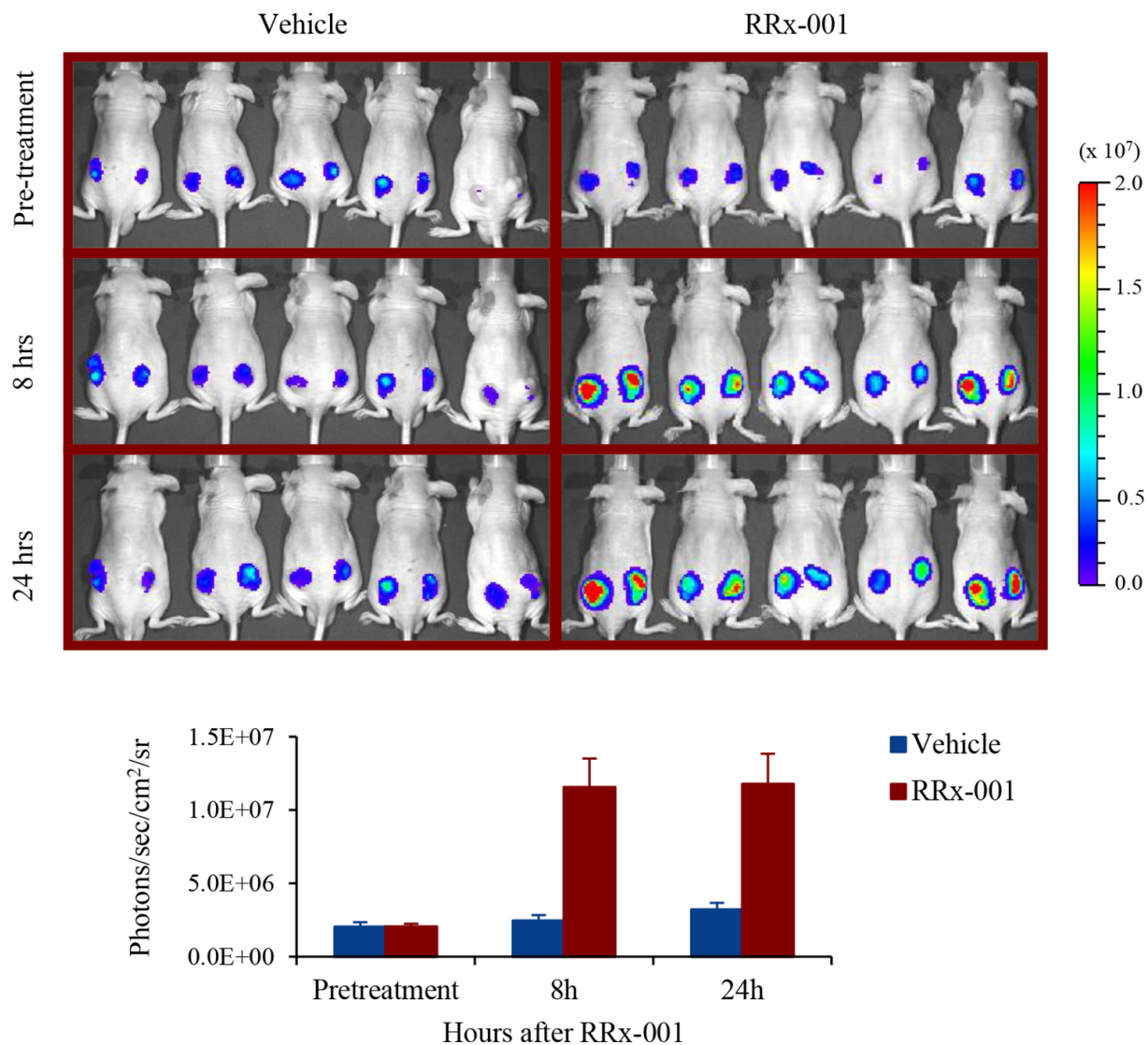

Figure 4: Optical bioluminescence imaging of ARE-FLUC activation by RRx-001 treatment in nude mice bearing SCC VII tumor xenograft. A. Bioluminescence signal measured $24 \mathrm{~h}$ before (Pre-treatment) and $8 \mathrm{~h}$ and $24 \mathrm{~h}$ after treatment with RRx-001 by IVIS optical bioluminescence imaging in vivo in animals. B. Quantitative graph showing the bioluminescence signal measured from image shown in (A). 
The ARE-FLUC/CMV-RLUC-mRFP expressing tumors grew as aggressively as the parental tumors in mice with a tumor volume quadrupling time (TVQT, 4 times its pretreatment volume) of approximately $3.0 \pm 0.3$ and $3.5 \pm$ 0.7 days for the parental and the ARE-FLUC/CMV-RLUCmRFP tumors, respectively (Supplemental Figure 2). There was no statistical difference in TVQT between the parental and the ARE-expressing tumors $(n=8, P=0.09)$. The result indicates that the ARE reporter transfection did not significantly alter the SCC VII tumor growth pattern.

Next, we studied the tumor response to RRx-001 treatment and analyzed the correlation between the tumor response and the ARE reporter gene expression before and after RRx-001 treatment. Mice were subcutaneously inoculated with $5 \times 10^{5}$ ARE-FLUC/CMV-RLUC-mRFP expressing SCC VII cells. Two tumors per animal were inoculated on the left and right lower flank. Ten days after tumor inoculation, mice with tumors of average size of $150 \mathrm{~mm}^{3}$ were selected and randomized into two groups with 8 tumors per group: 1) vehicle control and 2) RRx001 treatment group. Mice were imaged for ARE-FLUC expression $24 \mathrm{~h}$ before and after RRx-001 administration. Results showed that a single dose of $10 \mathrm{mg} / \mathrm{kg} \mathrm{RRx}-001$ inhibited tumor growth and produced a tumor volume quadrupling time (TVQT) of $5.7 \pm 1.3$ days compared to $3.3 \pm 0.7$ days of vehicle control group $(P<0.05)$. The linear regression analysis of the tumor growth delay (4X TGD, i.e. the difference between the TVQT of RRx-001-treated tumors compared to that of untreated control tumors) showed that the 4X TGD of each tumor was negatively correlated to its pre-treatment level of the ARE signal (Figure $5 ; R^{2}=-0.69, P=0.02$ ). A relatively low level of the pre-treatment ARE-FLUC signal correlated with improved response to RRx-001 compared to tumors with no pre-treatment signal; this same pattern of correlation occurred even among individual tumors within the same host mouse. The correlation analysis also showed that there were no correlations between posttreatment ARE-FLUC signals at $8 \mathrm{~h}-24 \mathrm{~h}$ and the tumor response to RRx-001 treatment $\left(R^{2}=0.2-0.4, P>0.05\right)$.
Taken together, the data suggest that the pre-treatment ARE expression, reflecting the basal level of Nrf2 activity, is a biomarker for early prediction of therapeutic response to pro-oxidant $\mathrm{RRx}-001$ treatment.

\section{DISCUSSION}

RRx-001 is a small molecular pan-epigenetic agent with ROS/RNS-generating properties that targets hypoxic tumor cells [11-13]. Antitumor activity has been demonstrated both in preclinical models and in patients with heavily pretreated refractory solid tumors [11, 14]. Apart from epigenetic alterations, RRx-001 acts via pleiotropic mechanisms including redox signaling and redox-induced dysregulation of many different signal pathways such as Nrf2, p53, PARP cleavage, HIF1 alpha, and G6PD activity [18]. RRx-001 also triggers p53 and p21 activity in response to double-stranded DNA breaks as well as deregulates cancer cellular energetics and metabolism [13]. In this study, we explored the impact of RRx-001 on Nrf2 activation in SCC VII tumor cells harboring the ARE-FLUC reporter vector. When ARE-FLUC-expressing SCC VII cells were treated with RRx-001, activated ARE-FLUC signal was clearly detected. The ARE-FLUC signal after exposure to $2.3 \mu \mathrm{M}$ RRx-001 was 2.3-fold higher than observed in untreated control cells, and equal to $80 \%$ of the signal achieved from cells treated with $20 \mu \mathrm{M}$ of TBHQ, a known Nrf2 activator.

$\mathrm{Nrf2}$ is a transcription factor that controls the expression of a set of phase II detoxifying enzymes such as NQO1, HO-1, GCLC, GST, UGT, and Mrp. Nrf2 signaling itself is regulated by a variety of mechanisms from the transcriptional control of the $\mathrm{Nrf} 2$ gene to activation of ARE by the Nrf2 complex. The Nrf2-Keap1 complex is the most important regulator of Nrf2 nuclear translocation through proteasomal degradation [19]. Keap1, an E3-ubiquitin ligase, targets Nrf2 for ubiquitin-dependent degradation, and is the major mechanism by which $\mathrm{Nrf2}$ nuclear levels are tightly controlled in cells. In addition to
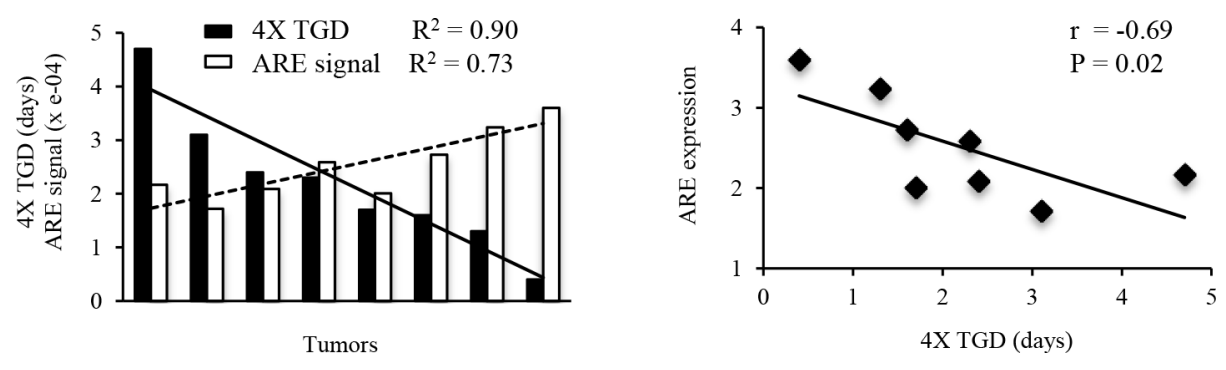

Figure 5: Correlation of pre-treatment ARE expression vs. tumor response to RRx-001 treatment. 
Keap1, Nrf2 degradation is also controlled by glycogen synthase kinase-3 (GSK-3)-mediated phosphorylation of two of its serine residues in the sequence DSGISL, which allows $\beta$-transducin repeat-containing protein $(\beta-\operatorname{TrCP})$ to ubiquitinate Nrf2 and promote its degradation [20]. In parallel to these proteins, many other proteins are identified as Nrf2 stabilizers either by directly binding with Nrf2, such as p21 Cip1/WAF1 [21], BRCA1 [22-23] and DJ-1 [24], or through indirect binding with Keap1 [25]. In oxidative stress, Nrf2 is phosphorylated and trafficked into the nucleus where it recruits its client protein smallmaf and binds to antioxidant response elements (ARE), leading to the coordinated induction of antioxidant genes. Similarly, through the release of ROS and RNS, RRx-001 activates Nrf2 with the subsequent induction of downstream target gene expression.

Nrf2 activity has been labeled a "double-edged sword" with both anti- and pro-tumorigenic properties, which can be both beneficial and deleterious: on the one hand, Nrf2 protects normal cells from oxidative stress and confers protection against tumorigenesis, while, on the other hand, it promotes ROS detoxification and tumor cell proliferation [7]. In general, the Nrf2-Keap1 pathway protects the cell and exerts a beneficial effect, delaying or preventing the onset of diseases like atherosclerosis, Alzheimer's disease, and rheumatoid arthritis [2]. However, the dark side of Nrf2 is that it also contributes to chemo- and radio-resistance of human cancers [15-17], since activation of the Nrf2-Keap1 pathway by anticancer therapeutics increases the intracellular antioxidant capacity and therefore not only protects cancer cells but may also accelerate their proliferation. In this context, Nrf2 inhibitors may help to overcome treatment resistance. Hence anticancer therapies may be made more effective if the mechanisms by which Nrf2 activators and/or inhibitors mediate the transition from anti- to pro-apoptotic effects are better understood. As demonstrated here, RRx-001 activates Nrf2-ARE signaling while exerting cytotoxicity by oxidative damage; however its effect in tumors with higher basal levels of activated Nrf2-ARE signaling is reduced as compared to tumors with lower activation.

Nrf2 regulates the production of mitochondrial and cytosolic ROS through NADPH oxidase [26] and therefore knockdown of Nrf2 provokes ROS accumulation and ultimately induces lethal DNA damage. As we reported here, genetic knockdown of the endogenous Nrf2 expression by Nrf2-specific siRNA increased the sensitivity of cancer cells to RRx-001. This result clearly suggests that in combination with Nrf2 inhibitors the anti-cancer properties of RRx-001 will be enhanced, while RRx-001-induced activation of Nrf2 may have beneficial effects on ROSmediated diseases like diabetes, rheumatoid arthritis and atherosclerosis. In fact, a number of chemically diverse Nrf2 activators have been identified and preclinically evaluated for therapeutic properties. Activation of Nrf2 by sulforaphane reduced hyperglycemia-induced stimulation of the hexosamine and PKC pathways and increased excretion of the glycating agent, methylglyoxal [27]. Cinnamic aldehyde, caffeic acid phenethylester and bardoxolone methyl, which similarly activate Nrf2, are under investigation for treatment of diabetes complications [28, $29]$. Beside these chemical compounds, varieties of natural compounds have also been identified as Nrf2 activators [30, 31]. However, all of the above activators, unlike RRx-001, lack apoptosis-inducing properties, and further research is needed to determine their therapeutic potential in other major diseases.

In summary, molecular imaging of ARE-FLUC and RLUC-mRFP co-expressing tumors in vitro and in tumor-bearing mice reveals that RRx-001 is a potent activator of the Nrf2-ARE signaling pathway via ROS/ RNS generation. The level of the pretreatment signal of ARE-FLUC in tumors is inversely correlated with the tumor response to RRx-001 treatment. Inhibition of endogenous Nrf2 expression by Nrf2-specific siRNA increases the cellular sensitivity to RRx-001 treatment. These results suggest that the basal level of Nrf2 and/or its downstream gene expression and/or genes expressed under ARE may serve as a biomarker for predicting therapeutic response of cancers to $\mathrm{RRx}-001$ treatment, and for selection and stratification of cancer patients who would be most likely to respond and benefit from RRx-001 therapy. Furthermore, the combined use of RRx-001 and Nrf2 inhibitors may enhance the anticancer efficacy and therefore this approach requires further study.

\section{MATERIALS AND METHODS}

\section{Materials}

RRx-001 was obtained from ATK Aerospace Systems [10]. The synthesis and characterization of RRx-001 is reported in detail elsewhere [8-12]. For in vitro cell culture experiments, $\mathrm{RRx}-001$ was dissolved in DMSO and then diluted with growth medium with a final concentration of DMSO at $<0.05 \%$. For animal experiments, RRx-001 formulation was prepared by dissolving $10 \mathrm{mg}$ RRx-001 in $0.5 \mathrm{~mL}$ DMA-PEG 400 $(1: 2)$ and then diluting with double distilled water to obtain a $2 \mathrm{mg} / \mathrm{mL}$ solution for injection.

The pcDNA-ARE-FLUC and pcDNACMV-RLUC-mRFP vectors were constructed in our lab. Nrf2-specific small interfering RNA (Nrf2-siRNA, sc-37049), scrambled siRNA (sc-37007), transfection reagents (sc-29528 and sc-36868), and antibodies against Nrf2 (sc-13032), HO-1 (sc-10789), NQO1 (sc-393736), B-actin (sc-130656) and lamin-B (sc-365962) were purchased from Santa Cruz Biotechnology, Santa Cruz, CA.

\section{Cell culture and transfection}

The SCC VII murine squamous cell carcinoma cells (32) were grown and maintained in DMEM 
medium (Invitrogen, Carlsbad, CA) supplemented with $10 \%$ fetal calf serum, 100 units $/ \mathrm{ml}$ penicillin, and $100 \mu \mathrm{g} / \mathrm{mL}$ streptomycin in a $37^{\circ} \mathrm{C}$ humidified incubator with a mixture of $95 \%$ air and $5 \% \mathrm{CO}_{2}$. The identity of cells has regularly been confirmed throughout the course of the studies by observation of the growth pattern and cell morphology in vitro and in vivo. All experiments were performed on exponentially growing cells with cell population doubling times of approximately $20 \mathrm{~h}$.

To silence the expression of endogenous Nrf2, SCC VII cells were transiently transfected with Nrf2-specific small interfering RNA (Nrf2-siRNA) by following the manufacturer's protocol. SCC VII cells were also transfected with a scrambled siRNA as a non-specific control. Briefly, SCC VII cells were seeded at a density of $1.0 \times 10^{6}$ cells/60-mm dish and transfected with either 120 pmol of Nrf2 siRNA or scrambled siRNA in siRNA transfection reagent-containing medium for $24 \mathrm{~h}$ at $37^{\circ} \mathrm{C}$. The siRNA-containing medium was then replaced with normal growth medium and incubated for an additional 24 h. Nrf2 expression and response to RRx-001 treatment were then assayed using Western blot and WST-8 assay (Dojindo Molecular Technologies, Santa Clara, CA) as previously reported [11].

To create a stable cell line co-expressing AREFirefly luciferase (ARE-FLUC) and CMV-Renilla luciferase-monomeric red fluorescent protein (RLUCmRFP) fusion proteins, cells at $80 \%$ confluency in $100-\mathrm{mm}$ plates were co-transfected with $10 \mu \mathrm{g}$ each of pcPUR-ARE-FLUC and pcDNA-CMV-RLUCmRFP plasmids by lipofectamine-mediated transfection (Supplemental Figure 3). Single clones of cells coexpressing both the reporter genes were double selected by puromycin and G418, and a colony was picked after bioluminescence imaging and sorted twice by FACS in RFP window. The expanded cells were evaluated by dualluciferase reporter assay for FLUC and RLUC reporter activities in vitro.

\section{In vitro dual-luciferase assay}

A dual-luciferase reporter assay was performed to verify the expression of FLUC and RLUC in transfected SCC VII tumor cells. Cells $\left(1 \times 10^{6}\right)$ were lysed in $200 \mu 1$ of passive lysis buffer (Promega, Madison, WI) by gently shaking for $10 \mathrm{~min}$ at room temperature, and the whole cell lysate was centrifuged at 10,000 rpm for $5 \mathrm{~min}$. The cleared supernatant of $20 \mu \mathrm{l}$ was mixed with $100 \mu \mathrm{l}$ of LARII solution (Promega) and measured for 10 seconds on a GloMax-20/20-luminometer (Promega). Similarly, a $20 \mu \mathrm{l}$ aliquot of lysate was mixed with $1 \mu \mathrm{g}$ of coelenterazine in $100 \mu \mathrm{l}$ of PBS for RLUC signal measurement by luminometer. The total protein content of each sample was used to normalize the results. The cells were also tested for the ARE-FLUC response by inducing with the known
Nrf2-activator tert-butylhydroquinone (TBHQ) and with $\mathrm{RRx}-001$ in various concentrations at different time points.

\section{Western blot analysis}

After exposure to RRx-001, cells were washed twice with cold PBS and lysed in RIPA buffer or NE-PER nuclear and cytoplasmic extraction buffer (Pierce Biotech, Rockford, IL) for extraction and separation of the cytosolic and nuclear proteins. For tumor samples, tumor-bearing mice were euthanized in a $\mathrm{CO}_{2}$ chamber at designated time points after RRx-001 treatment and perfused with $50 \mathrm{ml}$ PBS to remove blood cells. Tumors were homogenized in $20 \mathrm{mM}$ HEPES buffer (containing $1 \mathrm{mM}$ EGTA, $210 \mathrm{mM}$ mannitol and $70 \mathrm{mM}$ sucrose), centrifuged and the supernatants were used for Western blot. The protein contents were quantified using a Bio-Rad protein assay kit (Bio-Rad, Hercules, CA). Samples containing equal amounts of total protein $(20 \mu \mathrm{g})$ were resolved in 10\% SDS-PAGE gel and transferred onto PVDF membrane. The membrane was blocked with 5\% non-fat milk and probed with primary antibody and HRP-conjugated secondary antibody (Santa Cruz Biotechnology). The immunoreactive proteins were detected with ECL plus chemiluminescence detection reagents (Amersham Biosciences, GE Life Sciences, Pittsburgh, PA), and quantified by ImageJ program (v1.47, $\mathrm{NIH}$ ). The Western blot analyses were run at least twice, unless otherwise specifically indicated.

\section{Tumor model and therapy}

Nude mice, male, 7-8 weeks old and 20-25 grams in body weight, were purchased from Charles River Laboratories. Mice were acclimated under specific pathogen-free conditions in the Veterinary Service Center of Stanford University animal facilities for 3-5 days before starting each experiment, and sterilized food and water were available ad libitum. Mice were injected subcutaneously in the left and right lower flank with $5 \times 10^{5} \mathrm{SCC}$ VII tumor cells stably co-expressing ARE-FLUC and CMV-RLUCmRFP in $0.05 \mathrm{ml}$ Hank's solution. Two tumors were implanted per mouse. When tumors reached an average size of $150 \mathrm{~mm}^{3}$ (10 days after implantation), mice were randomly assigned to the treatment groups and imaged. RRx-001 was injected i.v. at doses as specified in each experiment. The tumor size was measured with calipers before treatment, and three times a week thereafter until the tumor volume reached at least 4 times $(4 \times)$ the pretreatment volume. The tumor volume was calculated using the formula: tumor volume $=\pi / 6 \times$ length $\times$ width $^{2}$. The tumor volume quadrupling time (TVQT, $4 \times$ )) was determined by a best-fit regression analysis. The tumor growth delay (TGD) is the difference between the TVQT of treated tumors compared to that of untreated control 
tumors. Both the TVQT and TGD were calculated for each individual animal, and then averaged for each group. The data are presented as percent $(\%)$ of the pretreatment volume on day 0 . Body weight of animals was measured three times a week. The animal experiments described herein were approved by the Stanford University Administrative Panel for Laboratory Animal Care.

\section{Bioluminescence imaging (BLI)}

A Xenogen IVIS 200 Imaging System was used in this study. Tumors were imaged for basal FLUC, RLUC, and mRFP signals $24 \mathrm{~h}$ before administration of RRx-001. Following injection with RRx-001, mice were imaged for RLUC signal by intravenous injection of $50 \mu \mathrm{g}$ of coelenterazine at 8 and $24 \mathrm{~h}$ post-RRx-001 treatment. Similarly, mice were imaged for FLUC signal $6 \mathrm{~h}$ after RLUC imaging by intraperitoneal injection of $3 \mathrm{mg}$ of D-Luciferin in $100 \mu \mathrm{l} \mathrm{PBS}$. The animals injected with vehicle (without RRx-001) served as the untreated control. The BLI signals over the region of interest (ROI) were quantified by Living Image software (Caliper Life Sciences, Alameda, CA). The BLI signals in RRx-001-treated tumors were quantitated and compared with the BLI signals of untreated control tumors. The mRFP signal was collected at all imaging sessions by excitation at $580 \mathrm{~nm} / \mathrm{emission}$ at $610 \mathrm{~nm}$.

\section{Statistics}

Data were statistically analyzed using a two-tailed Student's $t$-test. The correlation between the tumor response to RRx-001 treatment and the level of ARE signal was analyzed by a linear regression analysis.

\section{ACKNOWLEDGMENTS AND FUNDING}

This study was supported by EpicentRx, Inc research fund. EpicentRx greatly acknowledges the collaboration with ATK Aerospace Systems on the development of RRx-001.

\section{CONFLICTS OF INTEREST}

Jan Scicinski and Bryan Oronsky are employees of EpicentRx, Inc, and Susan Knox is a founder of EpicentRx, Inc.

\section{REFERENCES}

1. Mitsuishi Y, Motohashi H, Yamamoto M. The Keap1-Nrf2 system in cancer: Stress response and anabolic metabolism. Front Oncol. 2012; 2:200.
2. Itoh K, Mimura J, Yamamoto M. Discovery of the negative regulator of Nrf2, Keap1: a historical overview. Antioxid Redox Signal. 2010; 13:1665-78.

3. Bloom DA, Jaiswal AK. Phosphorylation of Nrf2 at Ser(40) by pro- tein kinase $\mathrm{C}$ in response to antioxidants leads to the release of $\mathrm{Nrf} 2$ from INrf2, but is not required for $\mathrm{Nrf} 2$ stabilization/accumulation in the nucleus and transcriptional activation of antioxidant response element-mediated $\mathrm{NAD}(\mathrm{P}) \mathrm{H}$ :quinine oxidoreductase-1 gene expression. J Biol Chem. 2003; 278:44675-82.

4. Huang F, Nie C, Yang Y, Yue W, Ren Y, Shang Y, Wang X, Jin H, Xu C, Chen Q. Selenite induces redoxdependent Bax activation and apoptosis in colorectal cancer cells. Free Radic Biol Med. 2009; 46:1186-96.

5. Hayes JD, Dinkova-Kostova AT. The Nrf2 regulatory network provides an interface between redox and intermediary metabolism. Trends Biochem Sci. 2014; 39:199-218.

6. Nguyen T, Sherratt PJ, Pickett CB. Regulatory mechanisms controlling gene expression mediated by the antioxidant response element. Annu Rev Pharmacol Toxicol. 2003; 43:233-60.

7. Lau A, Villeneuve NF, Sun Z, Wong PK, Zhang DD. Dual roles of Nrf2 in cancer. Pharmacol Res. 2008; 58:262-70.

8. Bednarski MD, Knox SJ, Cannizzo L, Warner K, Wardle R, Velarde S, Ning S. Cyclic nitro compounds, pharmaceutical compositions thereof and uses thereof. United States patent US7507842B2, 2009.

9. Straessler NA, Cannizzo LF, Li P, Kramer MP, Rosellberg DM. Methods of synthesizing and isolating $\mathrm{N}$-(bromoacetyl)-3'3-dinitroazetidine and a composition including the same. United States patent US8471041B2, 2013.

10. Straessler NA, Lesley MW, Cannizzo LF. Development of a Safe and Efficient Two-Step Synthesis for Preparing 1-Bromoacetyl-3, 3-dinitroazetidine, a Novel Clinical Anticancer Candidate. Org Process Res Dev. 2012; 16:512-7.

11. Ning S, Bednarski MD, Oronsky B, Scicinski J, Saul G, Knox SJ. Dinitroazetidines are a novel class of anticancer agents and hypoxia-activated radiation sensitizers developed from highly energetic materials. Cancer Res. 2012; $72: 2600-8$.

12. Scicinski J, Oronsky B, Taylor M, Luo G, Musick T, Marini J, Adams CM, Fitch WL. Preclinical evaluation of the metabolism and disposition of RRx-001, a novel investigative anticancer agent. Drug Metab Dispos. 2012; 40:1810-6.

13. Oronsky B, Oronsky N, Knox SJ, Fanger G, Scicinski J. Episensitization: therapeutic tumor resensitization by epigenetic agents: a review and reassessment. Anticancer Agents Med Chem. 2014; 14:1121-7.

14. Reid T, Dad S, Korn R, Oronsky B, Knox SJ, Scicinski J. Two Case Reports of Resensitization to Previous 
Chemotherapy with the Novel Hypoxia-Activated Hypomethylating Anticancer Agent RRx-001 in Metastatic Colorectal Cancer Patients. Case Rep Oncol. 2014; 7:79-85.

15. Wang XJ, Sun Z, Villeneuve NF, Zhang S, Zhao F, Li Y, Chen W, Yi X, Zheng W, Wondrak GT, Wong PK, Zhang D. Nrf2 enhances resistance of cancer cells to chemotherapeutic drugs, the dark side of Nrf2. Carcinogenesis. 2008; 29:1235-43.

16. Jiang T, Chen N, Zhao F, Wang XJ, Kong B, Zheng W, Zhang DD. High levels of Nrf2 determine chemoresistance in type II endometrial cancer. Cancer Res. 2010; 70:5486-96.

17. McDonald JY, Kim K, Norris AJ, Vlashi E, Phillips TM, Lagadec C, Della Donna L, Ratikan J, Szelag H, Hlatky L, McBride WH. Ionizing radiation activates the Nrf2 antioxidant response. Cancer Res. 2010; 70:8886-95.

18. Magesh S, Chen Y, Hu L. Small molecule modulators of Keap1-Nrf2-ARE pathway as potential preventive and therapeutic agents. Med Res Rev. 2012; 32:687-726.

19. Kobayashi A, Kang MI, Okawa H, Ohtsuji M, Zenke Y, Chiba T, Igarashi K, Yamamoto M. Oxidative stress sensor Keap1 functions as an adaptor for Cul3-based E3 ligase to regulate proteasomal degradation of $\mathrm{Nrf} 2$. Mol Cell Biol. 2004; 24:7130-9.

20. Rada P, Rojo AI, Evrard-Todeschi N, Innamorato NG, Cotte A, Jawarski T, Tobon-Velasco JC, Devijver H, Garcia-mayoral MF, Van Leuven F, Hayes JD, Bertho G, Cuadrado A. Structural and functional characterization of Nrf2 degradation by the glycogen synthase kinase $3 / \beta-\operatorname{TrCP}$ axis. Mol Cell Biol. 2012; 32:3486-99.

21. Chen W, Sun Z, Wang XJ, Jiang T, Huang Z, Fang D, Zhang DD. Direct interaction between Nrf2 and p21(Cip1/ WAF1) upregulates the Nrf2-mediated antioxidant response. Mol Cell. 2009; 34:663-73.

22. Gorrini C, Baniasadi PS, Harris IS, Silvester J, Inoue S, Snow B, Joshi PA, Wakeham A, Molyneux SD, Martin B, Bouwman P, Cescon DW, Elia AJ, Winterton-Perks Z, Cruickshank J, Brenner D, Tseng A, Musgrave M, Berman HK, Khokha R, Jonkers J, Mak TW, Gauthier ML. BRCA1 interacts with Nrf2 to regulate antioxidant signaling and cell survival. J Exp Med. 2013; 210:1529-44.

23. Gorrini C, Gang BP, Bassi C, Wakeham A, Baniasadi SP, Hao Z, Li WY, Cescon DW, Li YT, Molyneux S, Penrod N, Lupien M, Schmidt EE, Stambolic V, Gauthier ML, Mak TW. Estrogen controls the survival of BRCA1-deficient cells via a PI3K-NRF2-regulated pathway. Proc Natl Acad Sci USA. 2014; 111:4472-7.

24. Gan L, Johnson DA, Johnson JA. Keap1-Nrf2 activation in the presence and absence of DJ-1. Eur J Neurosci. 2010; 31:967-77.

25. Jiang ZY, Chu HX, Xi MY, Yang TT, Jia JM, Huang JJ, Guo XK, Zhang XJ, You QD, Sun HP. Insight into the intermolecular recognition mechanism between Keap1 and IKK $\beta$ combining homology modelling, protein-protein docking, molecular dynamics simulations and virtual alanine mutation. PloS One. 2013; 8:e75076.

26. Kovac S, Angelova PR, Holmstrom KM, DinkovaKostova AT, Abramov AY. Nrf2 regulates ROS production by mitochondria and NADPH oxidase. Biochim Biophys Acta. 2014; 850:794-801.

27. Xue M, Qian Q, Adaikalakoteswari A, Rabbani N, Babaei-Jadidi R, Thornalley PJ. Activation of NF-E2-related factor-2 reverses biochemical dysfunction of endothelial cells induced by hyperglycemia linked to vascular disease. Diabetes. 2008; 57:2809-17.

28. Lee Y, Shin DH, Kim JH, Hong S, Choi D, Kim YJ, Kwak MK, Jung Y. Caffeic acid phenethyl ester-mediated Nrf2 activation and IkappaB kinase inhibition are involved in NFkappaB inhibitory effect: structural analysis for NFkappaB inhibition. Eur J Pharmacol. 2010; 643:21-8.

29. Dinkova-Kostova AT, Liby KT, Stephenson KK, Holzclaw WD, Gao X, Suh N, Williams C, Risingsong R, Honda T, Gribble GW, Sporn MB, Talalay P. Extremely potent triterpenoid inducers of the phase 2 response: correlations of protection against oxidant and inflammatory stress. Proc Natl Acad Sci USA. 2005; 102:4584-9.

30. Wen Z, Wang Z, Wang S, Ravula R, Yang L, Xu J, Wang C, Zuo Z, Chow MS, Shi L, Huang Y. Discovery of molecular mechanisms of traditional Chinese medicinal formula Si-Wu-Tang using gene expression microarray and connectivity map. PloS One. 2011; 6:e18278.

31. $\mathrm{Hu} \mathrm{Q}$, Zhang DD, Wang L, Lou H, Ren D. Eriodictyol-7-O-glucoside, a novel Nrf2 activator, confers protection against cisplatin-induced toxicity. Food Chem Toxicol. 2012; 50:1927-32.

32. Fu KK, Lam KN, Rayner PA. The influence of time sequence of cisplatin administration and continuous low dose rate irradiation (CLDRI) on their combined effects on a murine squamous cell carcinoma. Int J Radiat Oncol Biol Phys. 1985; 11:2119-24. 